\title{
Malignant Acanthosis Nigricans Associated with Ovarian Cancer
}

\author{
Chee Won Oh ${ }^{\mathrm{a}}$ Jimi Yoon ${ }^{\mathrm{b}}$ Chi Yeon Kim ${ }^{\mathrm{b}}$ \\ aDepartment of Dermatology, School of Medicine, Kangwon National University \\ Hospital, Hyoja-dong, Chuncheon, and ' ${ }^{b}$ Department of Dermatology, School of \\ Medicine, Gyeongsang Institute of Health Science, Gyeongsang National \\ University and Hospital, Jinju, Korea
}

\section{Key Words}

Acanthosis nigricans - Ovarian cancer · Paraneoplastic dermatosis

\begin{abstract}
Malignant acanthosis nigricans is a cutaneous eruption characterized by symmetric hyperpigmented hyperkeratosis, dermal papillomatosis, and mucosal involvement associated with internal malignancies. Tripe palms refers to a characteristic velvety thickening of the palms, with exaggeration of normal skin markings. We present the second case of ovarian cancer in association with two coexisting paraneoplastic dermatoses occurring in a 57-year-old Korean female. The presence of acanthosis nigricans in conjunction with tripe palms in a female patient is highly suggestive of an internal malignancy including an ovarian cancer and demands an extensive search for the hidden ovarian cancer.
\end{abstract}

\section{Introduction}

Malignant acanthosis nigricans (MAN) is a cutaneous marker of internal malignancies. MAN has been known to be associated with intra-abdominal malignancies, most commonly gastric adenocarcinomas, along with tripe palms and the sign of Leser-Trélat. Unlike MAN associated with intra-abdominal malignancies, merely a few cases with MAN associated with gynecological malignancies have been reported. We present a case of MAN that was a manifestation of a paraneoplastic dermatosis of an ovarian cancer. The presence of sudden-onset MAN in conjunction with tripe palms may hint at gynecologic malignancies including ovarian cancers and necessitates extensive search for gynecologic cancers. 


\section{Case Report}

A 57-year-old woman presented with progressive hyperpigmentation and hyperkeratosis of 12 months' duration spreading from her upper extremities to the whole body. At first, she noted scattered skin tags on both forearms and thickened palms and soles. Minute physical examination revealed hyperpigmented velvety plaques on her neck, axillae, and groins, inflamed folds and whitish hyperplastic vegetations on the buccal mucosa and tongue, as well as multiple flat-topped papules on both forearms and coarse-thickened palms and soles. She presented with a diffuse velvety thickening and prominent ridges of the palms and soles (fig. 1). Well-defined thickened brownish plaques in the axillary area were observed (ig. 2). She had no systemic symptoms such as general fatigue, weight loss or melena. The past medical and family history provided no significant information. A skin biopsy of the hyperkeratotic plaque on her palms showed marked hyperkeratosis and acanthosis in the epidermis (fig. 3). Histopathologic examination of the plaque in the axillae revealed hyperkeratosis, slightly irregular acanthosis and prominent dermal papillomatosis, which were compatible with the histopathologic findings of acanthosis nigricans (i․ 4 ). The patient was started on etretinate $20 \mathrm{mg}$ daily for 2 weeks for symptomatic control, and she was referred to an oncologist for an extensive examination for an underlying malignancy. The laboratory findings including $\mathrm{CBC}$, liver function test, urinary function test, thyroid function test and serum protein electrophoresis test were all within the normal range. The radiologic findings of the lung, heart and skeletal scintigraphy showed no evidence of malignancy. Colonoscopy and esophagogastroduodenoscopy did not reveal any evidence of gastrointestinal tract malignancy. AFP ( $\alpha$-fetoprotein), CEA (carcinoembryonic antigen), and CA 19-9 (carbohydrate antigen 19-9) were all within normal ranges, but the level of CA 125 (132 U/ml; normal range $<35 \mathrm{U} / \mathrm{ml}$ ) was elevated. Computed tomography (CT) and ultrasound of the abdomen were promptly performed and they revealed a $13.6 \mathrm{~cm}$ sized cystic and solid mass in the uterus (fig. 5 ). There were multiple paraaortic and retroperitoneal lymph node enlargements (fig. 5). She was diagnosed as stage IIIc ovarian carcinoma. The patient underwent exploratory laparotomy, total abdominal hysterectomy, bilateral salpingo-oophorectomy and periaortic lymph node sampling. In pathological examination, the mass turned out to be a serous adenocarcinoma. The patient was started on combination chemotherapy with paclitaxel and carboplastin, and her skin lesions gradually resolved after the third cycle of chemotherapy. After completing the sixth cycle of chemotherapy, her malignancy was in remission and she remained well with no aggravation and recurrence of the cancer and skin lesions for 2 years, except for mild hyperkeratosis of her axillae.

\section{Discussion}

MAN is a cutaneous eruption characterized by symmetric hyperpigmented hyperkeratosis, dermal papillomatosis, and mucosal involvement [1]. The most commonly involved locations are the axillae, neck, external genitalia, groin, face, inner thighs, perianal area, and antecubital and popliteal fossa [1]. MAN often accompanies tripe palms characterized by velvety thickening of the palms and exaggeration of the palmar ridges. As supported by numerous cases in the literature, the progressive form of acanthosis nigricans has been considered as a paraneoplastic dermatosis of intraabdominal malignancies along with tripe palms $[2,3]$. Various intra-abdominal malignancies such as gastric, esophageal, pancreatic, and hepatic duct and cyst adenocarcinomas have been associated with MAN [4]. In comparison to intra-abdominal malignancies, MAN in conjunction with extra-abdominal malignancies, especially gynecological malignancies, is relatively rare. Based on the literatures, 6 cases of endometrial carcinoma [5, 6], 1 case [4] of squamous cell carcinoma of the cervix, 3 cases of ovarian cancer $[4,7,14]$, and 1 case of uterine cancer [4] have been reported to occur in conjunction with MAN.

Among these, ovarian cancer is the most common cause of death resulting from a gynecological tumor. In the United States, an estimated 21,650 new cases and 15,520 deaths were projected for the year 2008 [8]. Ovarian cancer accounts for about 3\% of all cancers among women and is the second most common gynecologic malignancy, next to endometrial carcinoma. Since clinical symptoms at early stages are rare and are often 
mistaken for menopausal ailments or intestinal illnesses, the diagnosis is often made at advanced tumor stages with an overall 5-year survival rate of about $28 \%$ [9]. However, survival rates of greater than $80 \%$ have been reported with early-stage cancers, thus stimulating efforts to determine the role of population screening for the detection of early disease [10]. The survival rate of all patients with invasive epithelial ovarian cancer on screening (in the Shaaban and Rezvani group) was $89.9 \%$ at 2 years and $77.2 \%$ at 5 years, which was significantly higher than the survival rate of patients with ovarian cancer in the control group who did not have screening (2-year survival rate, 70.9\%; 5-year survival rate, $48.7 \%$ ) [10].

Therefore, identifying paraneoplastic dermatoses of ovarian cancer and detecting them earlier may play a role in screening procedures such as bimanual examination, ultrasonography, and measurement of serum tumor markers. Besides acanthosis nigricans, the paraneoplastic manifestations of ovarian cancer include paraneoplastic pemphigus, acquired ichthyosis, lupus-like syndrome, scleroderma and erythema annulare, digital necrosis, edema, and dermatographism [8]. In addition to the above mentioned dermatoses, recognition of the features and existence of a prodrome of ovarian carcinoma is important, since prodromal symptoms may manifest in conjunction with paraneoplastic skin changes. In general, a third to half of the patients developing ovarian cancer note recurrent abdominal bloating and pain, urinary symptoms or fatigue or malaise 3 months before their carcinoma is diagnosed [8].

The pathogenesis of MAN has yet to be determined; however, a few hypotheses have been suggested. A humoral factor produced by the tumor is likely, since there are cases in which the skin disorder improved or resolved following treatment of the malignancy [11]. Elevated urinary transforming growth factor- $\alpha$ (TGF- $\alpha$ ) and increased expression of epidermal growth factor (EGF) receptors in lesional skin were noted in a patient with acanthosis nigricans and Leser-Trélat sign [11]. A separate case with gastric cancer was also shown to express TGF- $\alpha$ and EGF receptors [12]. More recently, a pathogenic role has been proposed for a tyrosine kinase receptor expressed in the basal cells of the epidermis [13]. However, the specific humoral factor produced by tumors has not yet been reported in a separate case of ovarian cancer.

Early detection of ovarian cancer increases possibilities of successful treatment and survival rate. MAN can provide a clue to an early detection of ovarian cancers. Indeed, it has often been reported as the first sign of a malignancy that was discovered several months later. In the cases previously reported in the literature, acanthosis nigricans in one patient and tripe palms in another patient preceded the discovery of endometrial cancer by 4 years and 3 years, respectively [6]. Furthermore, the persistence or recurrence of skin lesion can be a significant indicator of successful treatment and neoplasm progression [5]. MAN tends to be parallel to the course of the cancer, namely, it will spread and worsen with progression of the tumor, regress with successful therapy, and recur with recurrence and metastases of the tumor [14-16]. 
Fig. 1. The patient presented with a diffuse velvety thickening and prominent ridges of the palms and soles.

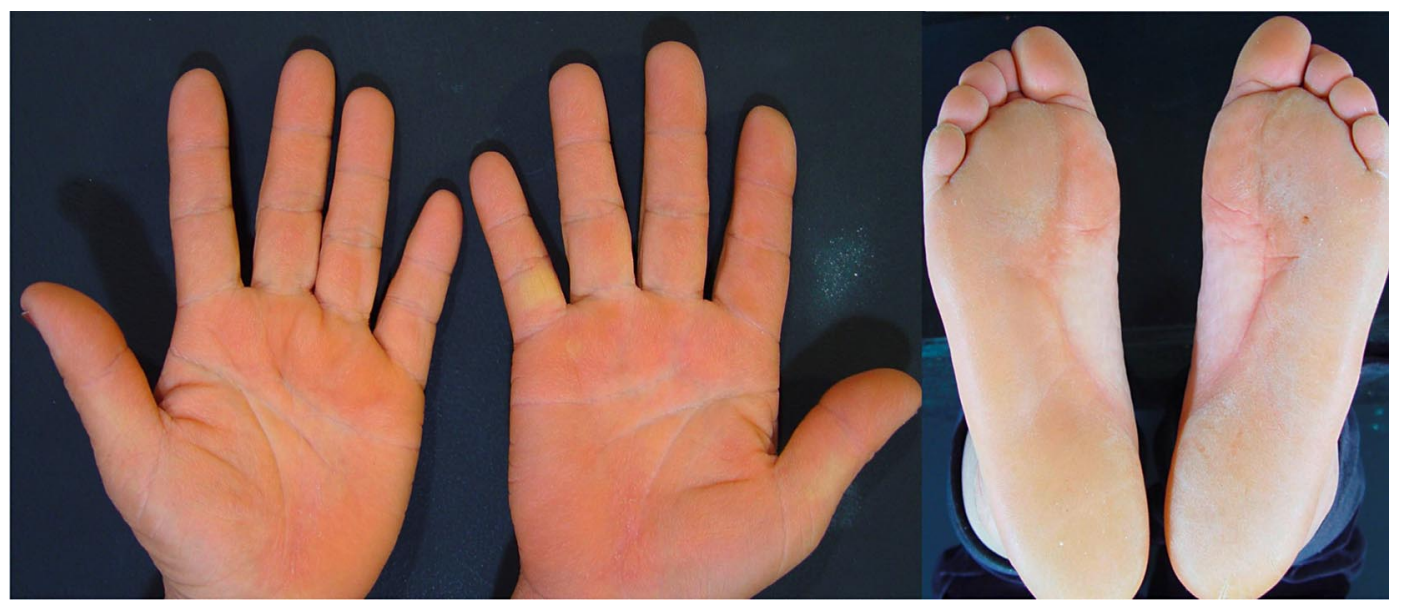

Fig. 2. Well-defined thickened brownish plaques on the axillary area were observed.

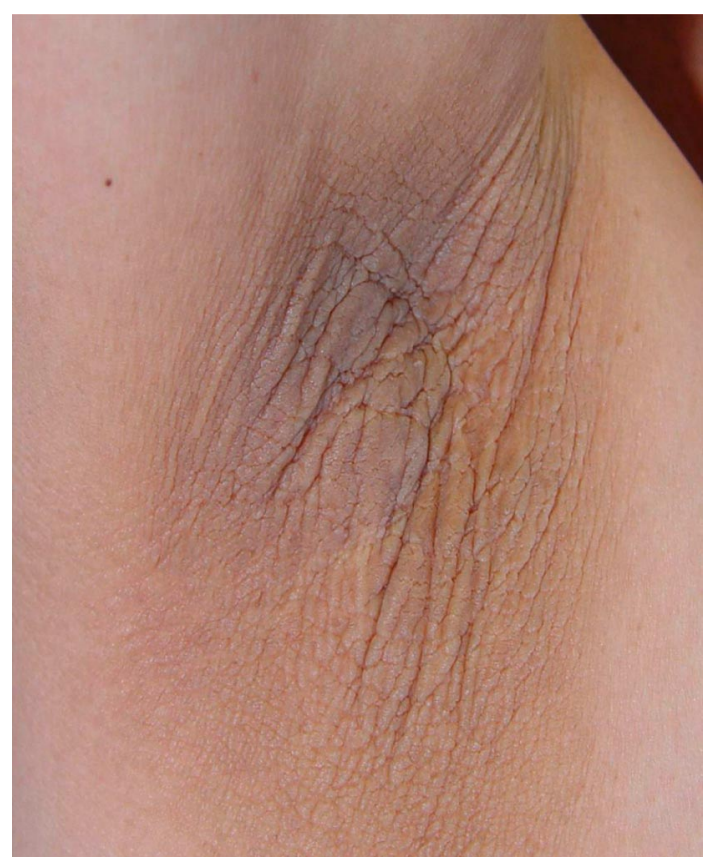


Fig. 3. A skin biopsy of the hyperkeratotic plaque of the palm showed marked hyperkeratosis and acanthosis in the epidermis (HE, 100×).

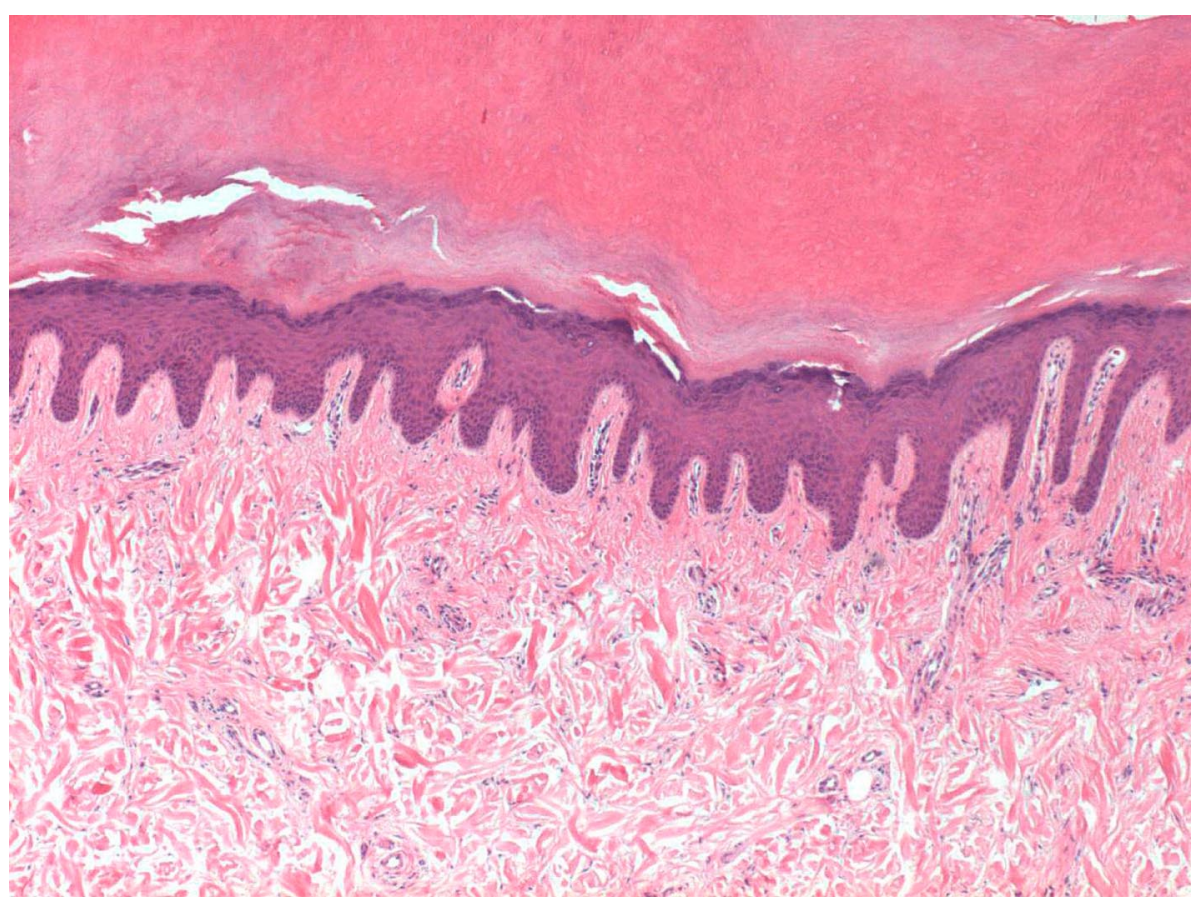

Fig. 4. Histopathologic examination of the plaque in the axillae revealed hyperkeratosis, slightly irregular acanthosis and prominent dermal papillomatosis (HE, 100×).

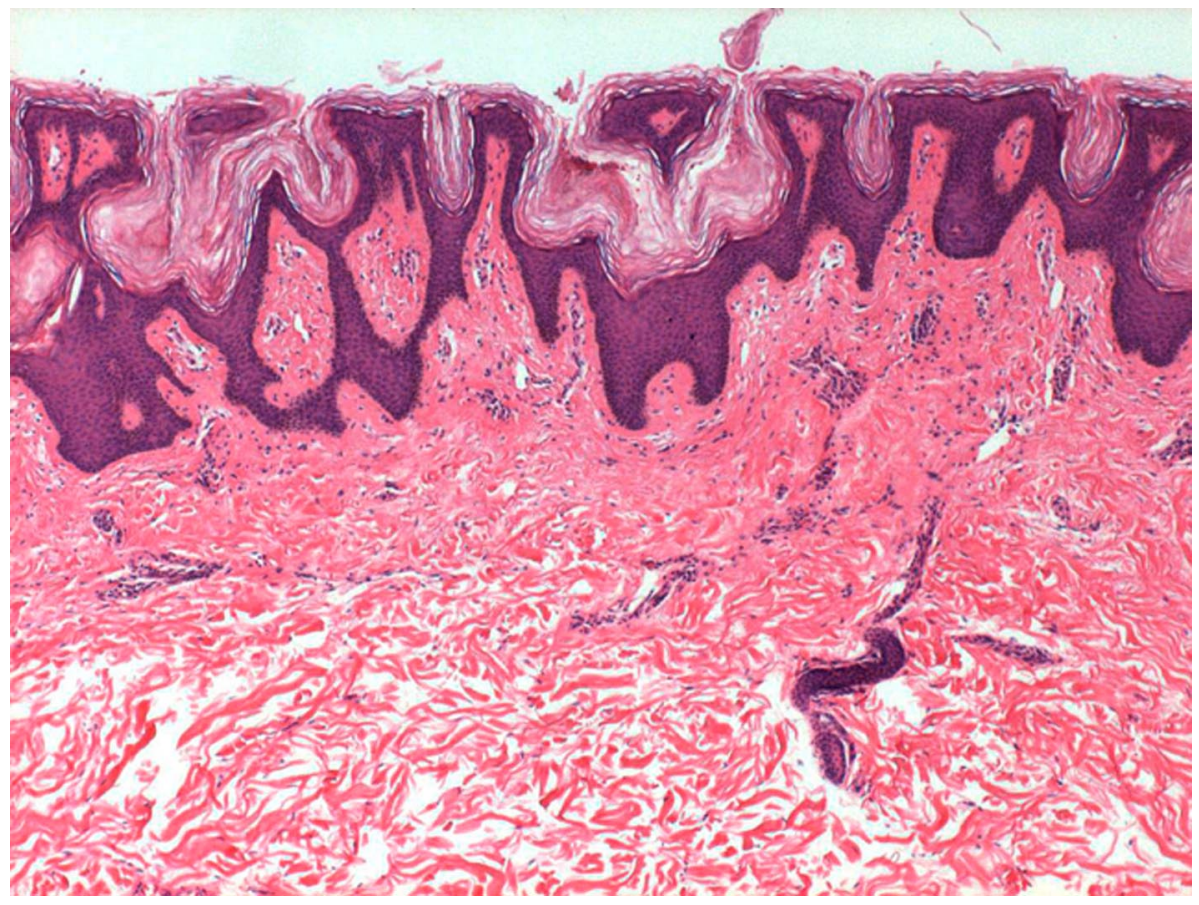


Fig. 5. CT revealed a $13.6-\mathrm{cm}$ sized cystic and solid mass in the uterus. There were multiple paraaortic and retroperitoneal lymph node enlargements.

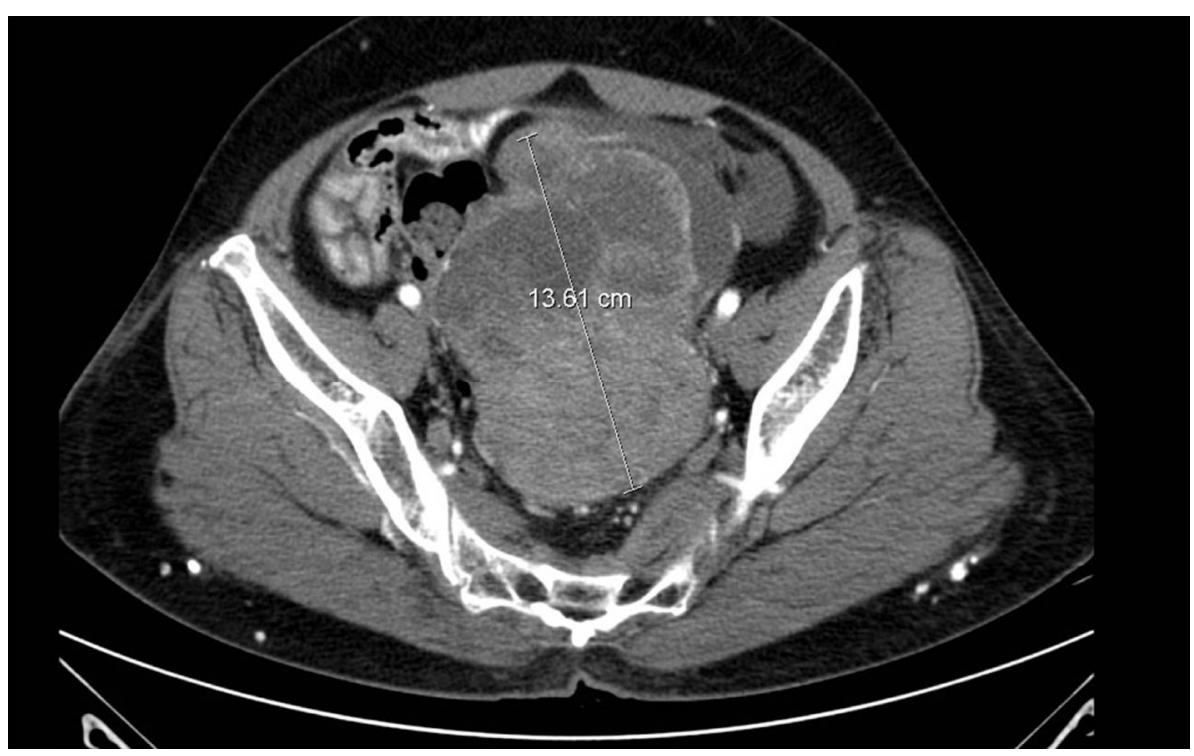




\section{References}

1 Schwartz RA: Acanthosis nigricans. J Am Acad Dermatol 1994;31:1-19.

-2 Stieler W, Plewig G: Acanthosis nigricans maligna and Leser-Trélat sign in double malignancy of the breast and stomach. Z Hautkr 1987;62:344-366.

-3 Yeh JS, Munn SE, Plunkett TA, Harper PG, Hopster DJ, du Vivier AW: Coexistence of acanthosis nigricans and the sign of Leser-Trélat in a patient with gastric adenocarcinoma: a case report and literature review. J Am Acad Dermatol 2000;42:357-362.

-4 Curth HO, Hilberg AW, Machacek GF: The site and histology of the cancer associated with acanthosis nigricans. Cancer 1962;15:364-382.

5 Gorisek B, Krajnc I, Rems D, Kuheli J: Malignant acanthosis nigricans and tripe palms in a patient with endometrial adenocarcinoma: a case report and review of the literature. Gynecol Oncol 1997;65:539-542.

-6 Tarek MM, Maurie M: Acanthosis nigricans with endometrial carcinoma: case report and review of the literature. Gynecol Oncol 2002;84:332-334.

7 Kebria MM, Belinson J, Kim R, Mekhail TM: Malignant acanthosis nigricans, tripe palms and the sign of Leser-Trélat, a hint to the diagnosis of early stage ovarian cancer: a case report and review of the literature. Gynecol Oncol 2006;10:353-355.

8 Scheinfeld N: A review of the cutaneous paraneoplastic associations and metastatic presentations of ovarian carcinoma. Clin Exp Dermatol 2007;33:10-15.

9 Quirk JT, Natarajan N: Ovarian cancer incidence in the United States, 1992-1999. Gynecol Oncol 2005;97:519-523.

10 Akram S, Mary R: Ovarian cancer: detection and radiologic staging. Clin Obstet Gynecol 2009;52:73-93.

11 Ellis DL, Kafka SP, Chow JC, et al: Melanoma, growth factors, acanthosis nigricans, the sign of Leser-Trélat, and multiple acrochordons: a possible role for alpha-transforming growth factor in cutaneous paraneoplastic syndromes. N Engl J Med 1987;317:1582-1587.

12 Wilgenbus K, et al: Further evidence that acanthosis nigricans maligna is linked to enhanced secretion by the tumour of transforming growth factor alpha. Arch Dermatol Res 1992;284:266-270.

13 Logié A, Dunois-Lardé C, Rosty C, et al: Activating mutations of the tyrosine kinase receptor FGFR3 are associated with benign skin tumors in mice and humans. Hum Mol Genet 2005;14:1153-1160.

14 Cohen PR, Grossman ME, Almeida L, Kurzrock R: Tripe palms and malignancy. J Clin Oncol 1989;7:669-678.

15 Anderson SH, Hudson-Peacock M, Muller AF, et al: Malignant acanthosis nigricans: potential role of chemotherapy. Br J Dermatol 1999;141:714-716.

16 Requena L, Aguilar A, et al: Tripe palms: a cutaneous marker of internal malignancy. J Dermatol 1995;22:492-495. 